\title{
Simultaneous infection of Schistosoma mansoni and S. rodhaini in Biomphalaria glabrata: impact on chronobiology and cercarial behaviour
} Alice Norton ${ }^{1,2}$, David Rollinson*2, Louisa Richards ${ }^{1}$ and Joanne Webster ${ }^{1}$

Address: ${ }^{1}$ Department of Infectious Disease Epidemiology, Imperial College Faculty of Medicine, London, W2 1PG, UK and ${ }^{2}$ Wolfson Wellcome Biomedical Laboratories, Department of Zoology, The Natural History Museum, London, SW7 5BD, UK

Email: Alice Norton - alice.rafferty03@imperial.ac.uk; David Rollinson* - D.Rollinson@nhm.ac.uk; Louisa Richards - richards.louisa@gmail.com; Joanne Webster - joanne.webster@imperial.ac.uk

* Corresponding author

Published: 2 December 2008

Parasites \& Vectors 2008, I:43
Received: 23 July 2008

Accepted: 2 December 2008

(C) 2008 Norton et al; licensee BioMed Central Ltd.

This is an Open Access article distributed under the terms of the Creative Commons Attribution License (http://creativecommons.org/licenses/by/2.0), which permits unrestricted use, distribution, and reproduction in any medium, provided the original work is properly cited.

\begin{abstract}
Background: The chances of a schistosome cercaria encountering a suitable definitive host may be enhanced by emergence from the molluscan intermediate host with maximal glycogen stores and by an appropriate chronobiological rhythm. This study aimed to identify and characterize the effects of potential competitive interactions in the snail host Biomphalaria glabrata, between the closely-related Schistosoma mansoni and S. rodhaini, on phenotypic behavioural traits. It was predicted that inter-specific competition would affect chronobiological emergence rhythms and reduce the activity of schistosome swimming behavioural traits. Biomphalaria glabrata snails (I20) were exposed to either $S$. mansoni or S. rodhaini single infections, or a mixed infection of both species simultaneously and the resulting cercarial phenotypic traits were characterised. Cercariae were identified from co-exposed snails by amplification and sequencing of the mitochondrial cytochrome oxidase subunit I (COI).

Results: S. mansoni and S. rodhaini largely maintained their distinct chronobiological rhythms after mixed exposures and infections. However, inter-specific competition appeared to result in a restriction of the shedding pattern of $S$. rodhaini and slight shift in the shedding pattern of S. mansoni. Inter-specific competition also significantly lowered hourly cercarial production for both parasite species in comparison to single exposures and infections and reduced cercarial swimming activity.

Conclusion: Inter-specific competition was shown to influence cercarial production, chronobiology and activity and should therefore be investigated further in field situations to determine the effects of these changes on parasite fitness (incorporating both host finding and infectivity) where these two species overlap. Importantly this competition did not result in a large change in chronobiological emergence of cercariae for either species indicating that it would not have a large influence on the species of hosts available for infection at time of emergence. This study has furthermore demonstrated the potential for phenotypic measures to provide markers for species-specific identification even in conditions of co-infection.
\end{abstract}




\section{Background}

Transmission between hosts is a vital part of the life-cycle for any parasitic organism. For many parasitic platyhelminths such transmission is achieved by free-living larval stages, which have limited energy reserves and short life-spans. Strong selection pressures are therefore expected to influence the phenotypic behaviour of such larvae in order to improve their transmission success.

Schistosomes of the genus Schistosoma are the causative agent of schistosomiasis, a parasitic disease second only to malaria in terms of its socio-economic and public health importance [1]. They are platyhelminth macroparasites with an indirect life-cycle involving a mammalian and molluscan host. Transmission between the two occurs via free swimming larval stages: cercariae (infective to the mammalian definitive host) and miracidia (infective to the molluscan intermediate host). Schistosome cercariae have a well developed tail with longitudinal striated muscles extending underneath the tegument with which they swim to find their definitive hosts. Glycogen stores within this tail provide a non-renewable energy source which is depleted during swimming [2]. Cercariae are therefore adapted to maximise their chance of encounter with a host by, for instance, emerging in the correct 'host-time' as well as 'host-space' and such adaptations may be specific to their definitive host species [2-4]. Cercarial swimming behaviours have been shown to be influenced by several stimuli such as light levels and host chemical signals $[5,6]$ Biotic pressures, for example competition from other species of parasites for shared resources within the same host, are also be predicted to reduce the glycogen reserves [7] and hence subsequent activity and infectivity of those cercariae shed $[7,8]$.

S. mansoni and S. rodhaini are closely related species within the lateral-spined S. mansoni group [9]. Their larval stages are morphologically similar and therefore difficult to distinguish visually. Furthermore, their host ranges may overlap in both the intermediate host (freshwater snails of the genus Biomphalaria) and the definitive host. S. mansoni can infect both primates and rodents [10] and $S$. rodhaini can infect rodents and canines $[11,12]$ and they are known to co-occur and co-infect in parts of Africa [1316]. Their main definitive hosts, however, differ being humans for $S$. mansoni and rodents for $S$. rodhaini. The cercarial behavioural repertoire of these two species may therefore be expected to differ to enhance transmission to these contrasting definitive hosts. Indeed, S. mansoni cercariae have been demonstrated by several authors to exhibit a diurnal shedding pattern and S. rodhaini cercariae a nocturnal shedding pattern therefore linking the timing of cercarial emergence in nature to coincide with the highest activity of their main definitive host species in the water $[12,13,17]$. Interestingly, a recently published field study reported, that $S$. rodhaini from Kenya can exhibit a bimodal cercarial emergence profile, with peaks between both 0500 and 0800 and also between 1900 and 2200 [16].

Under conditions where these two parasite species interact and compete, such interactions are predicted to influence their behaviour. The chronobiology of laboratorybred hybrids of these two species has previously been investigated [17] as well as intra-specific hybrids of two strains of $S$. mansoni with different shedding peaks from Guadeloupe [18] and these have both shown chronobiology to be genetically determined. Furthermore, the chronobiology of S. mansoni and Ribeiroia marini cercariae emerging from co-infected Biomphalaria glabrata [19] and $S$. haematobium and $S$. bovis cercariae emerging from coinfected Bulinus truncatus [20] have been investigated and small shifts in the parasite's chronobiology have been found. The aforementioned recent field study, which investigated the chronobiology of S. mansoni and S. rodhaini emerging from one co-infected snail, reported that co-infection did not affect either species' cercarial emergence patterns when measured phenotypically [16]. However, data from adult worms obtained by the infection with these cercariae of mice indicated that there may be an effect of co-infection on $S$. rodhaini's emergence [16]. No previous study has, however, been able to identify emerging cercariae to species using molecular techniques, meaning that for time periods where cercarial shedding of the two species overlap effects of competition may not have been accurately identified.

The main aim of this study was to use new molecular techniques [21] to accurately characterize the impact of any inter-specific competition between these species on cercarial emergence and to observe the impact on swimming activity. The elucidation of such behavioural repertoires is important for understanding the mechanisms of host location for parasites in general as phenotypic plasticity may be vital in determining a parasite's ability to infect the next host stage.

\section{Methods \\ Host-parasite maintenance}

Parasites used in these experiments were well established strains of $S$. mansoni and $S$. rodhaini which had been passaged routinely in the laboratory through B. glabrata (a species well adapted for laboratory culture and closely related to the natural Biomphalaria spp. hosts of the parasite species, the latter of which are not well adapted for laboratory culture and provide prohibitively small sample sizes for experimental models). All snails were maintained in the laboratory at $23-25^{\circ} \mathrm{C}$ and subjected to a 12 L (07:00-19:00): 12 D (19:00-07:00) light regime (full ultra-violet spectrum, Sun-glo natural sunlight lamps). 
Snails were housed individually in plastic pots $(10 \mathrm{~cm} \times 8$ $\mathrm{cm} \times 5 \mathrm{~cm}$ ) in $100 \mathrm{ml}$ of Caledonian spring water (Iceland plc., UK) changed weekly. All snails were fed ad libitum on fresh lettuce supplemented with fish food (Tetra Ltd) and chalk.

\section{Experimental design}

Four groups of 30, approximately $10 \mathrm{~mm}$ in width and sexually mature (as measured by onset of egg laying), $B$. glabrata were exposed to either $6 \mathrm{~S}$. mansoni miracidia, $6 \mathrm{~S}$. rodhaini miracidia, 6 miracidia of both species simultaneously or 3 miracidia of both species simultaneously. Individual exposure was performed in $5 \mathrm{ml}$ of spring water for two hours, a period sufficient for maximal miracidial penetration [22].

\section{Chronobiology}

At week seven post exposure of snails to miracidia, the chronobiology of the emerging cercariae was measured. Up to 20 randomly-selected shedding snails from each of the treatment groups were placed in individual vials of 16 $\mathrm{ml}$ artificial spring water [23] for $24 \mathrm{hrs}$ in the normal 12 L (07:00-19:00):12 D (19:00-07:00) light regime. The water was replaced every two hours and the number of cercariae shed every two hours estimated from the number of cercariae present in four $0.2 \mathrm{ml}$ samples. Those cercariae shed from snails exposed to both $S$. mansoni and $S$. rodhaini were pelleted by centrifugation and stored in ethanol at $-20^{\circ} \mathrm{C}$ for each snail at each time point for subsequent molecular sequencing and identification as described below.

\section{Cercarial swimming behaviour}

At weeks five and seven post initial parasite exposure cercariae were shed for two hours in the light (07:00-09:00) and then on a separate day for two hours in the dark (19:00-21:00) to allow for the production of both S. mansoni and S. rodhaini respectively. At both 09:00 and 21:00 cercariae from individual snails were videoed. This provided six different groups of cercariae from the four snail groups, as those snails which were co-exposed to both $S$. mansoni and S. rodhaini were shed at two different time points to allow the shedding of cercariae from both species. A minimum of four randomly selected cercariae (ranging from four to seven) from one snail were placed on a petri dish in $0.15 \mathrm{ml}$ water. They were videotaped (using an Olympus SZ4045 trinocular dissecting microscope attached to a JVC TK-1481 composite colour video camera) for five minutes. Four replicates with new cercariae from each snail were recorded at each time (07:00 and 19:00) on both weeks.

Behavioural categories used for recording were expanded from those described in Norton et al., 2007 [5] for S. mansoni to include new behaviours observed and further details on behavioural states where the cercariae were attached to the petri dish (see Table 1). Those behavioural categories performed whilst cercariae were unattached were numerically coded and those performed whilst the cercariae were attached were alphabetically coded. Continuous sampling within two second intervals was performed, with viewing order randomised and line identity hidden from the primary observer in order to avoid observer bias. Whilst this method of recording behavioural categories may not accurately reflect the natural environment (where cercariae mainly swim vertically in the water column), previous research has, however, demonstrated such measures to be useful in determining the activity levels of cercariae [7].

\section{Molecular analyses}

Cercarial pellets stored in ethanol were transferred onto Whatman FTA ${ }^{\circledast}$ cards for DNA preparation carried out according to the manufacturer's protocol Whatman FTA ${ }^{\circledast}$ cards. Polymerase chain reaction (PCR) amplifications were performed on a Geneamp PCR System 2700 (Applied Biosystems). Amplification of the mitochondrial cytochrome oxidase subunit 1 (CO1) was performed using 'ASMIT 1' (5'-3' TTTTTTGGGCATCTGAGGTTTAT) and 'ASMIT 2' (5'-3' TAAAGAAAGAACATAATGAAAATG) primers [24]. Amplifications were performed in $50 \mu \mathrm{l}$ reactions containing one plug of Whatman FTA $^{\circledast}$ card extracted DNA, $1 \mu \mathrm{l}$ of each primer (ASMIT 1 and ASMIT 2) at 50 pmolar concentration, $25 \mu \mathrm{l}$ of HotStarTaq Master Mix (Qiagen) and $23 \mu \mathrm{l}$ of $\mathrm{dH}_{2} \mathrm{O}$. Thermal cycling was performed under the following conditions: $15 \mathrm{~min}$ at $95^{\circ} \mathrm{C}$ to activate the HotStarTaq DNA Polymerase and for denaturation, followed by 45 cycles of $15 \mathrm{sec}$ at $94^{\circ} \mathrm{C}, 30 \mathrm{sec}$ at $40^{\circ} \mathrm{C}$ and $45 \mathrm{sec}$ at $72^{\circ} \mathrm{C}$; followed by a final 7 min extension at $72^{\circ} \mathrm{C}$. PCR products were purified in 96 well plates using a Millipore Montage PCR purification kit as per manufacturer's protocol. Sequencing was carried out using a BigDye Terminator v1.1 cycle sequencing kit (Applied Biosystems) in $10 \mu \mathrm{l}$ reactions containing $3 \mathrm{ng}$ of DNA for every 100 bps of PCR product, 1 pMols primer, 3 $\mu \mathrm{l}$ Big Dye dilution buffer $(2.5 \times), 1 \mu$ l Big Dye reaction mix and $\mathrm{x} \mu \mathrm{l} \mathrm{dH}_{2} \mathrm{O}$. Thermal cycling was performed under the following conditions: 5 min pre-denaturation at $96^{\circ} \mathrm{C}$ without Big Dye reaction mix, pause of cycler whilst samples transferred to ice and Big Dye added. Then 25 cycles of 10 secs were performed at $96^{\circ} \mathrm{C}, 5$ secs at $50^{\circ} \mathrm{C}$, and 4 mins at $60^{\circ} \mathrm{C}$. Sequences were imported into Sequencher vs. 3.1.1. (GeneCodes corp.) and compared to control sample sequences in order to identify the species of schistosome cercariae present in each sample. Cercarial samples containing both species were identified by the presence of double peaks at nucleotide positions that differed between $S$. mansoni and $S$. rodhaini in the CO1 sequence. 
Table I: Cercarial behavioural categories.

\section{Unattached behaviours}

I Straight-line- fast tail movements resulting in a linear progressive motion. Movement is tail first with the body following precisely in the direction of motion and tail undulating. Random changes in the direction of the motion occur without pause.

2 Zig- zag line- fast tail movements resulting in a linear progressive motion with constant changes in direction creating a zigzag pattern. Movement is tail first with the position of the body and tail undulating perpendicularly to the direction of motion. Random changes in the direction of motion occur without pause.

3 Circular line- fast tail movements resulting in a progressive motion. Movement is tail first with the body following precisely in the direction of motion. The progressive motion results in movement in a definite circle of measurable diameter with no random direction changes.

4 Spin- body and tail spin around the central axis of the cercaria whilst unattached. There is no linear movement

5 Corkscrew-body and tail spin around the central axis of the cercaria whilst unattached resulting in a linear progressive motion. The manner of spinning can be described as a 'corkscrew' due to the pattern formed by the forward motion. Random changes in the direction of motion occur without pause.

6 Drift- there is no motion of cercaria lasting for more than a brief second, where the cercaria appears to drift unattached.

4/6 Drifting with intermittent spins- there is no motion of cercaria lasting for more than a brief second, where the cercaria appears to drift unattached. This is interrupted by spins where the body and tail rotate around the central axis of the cercaria resulting in a tight spin whilst unattached. There is no linear movement

1/6 Drifting with intermittent forward motion- there is no motion of cercaria lasting for more than a brief second, where the cercaria appears to drift unattached. This is interrupted by fast tail movements resulting in a linear progressive motion. Movement is tail first with the body following precisely in the direction of motion and tail undulating. Random changes in the direction of the motion occur without pause.

7 Body first- progressive motion using the body first with the tail following in the direction of motion. Random changes in direction which occur without pause.

\section{Attached behaviours}

\section{A Attached, but no motion}

T Slow tail movements from side to side or curling with body as point of attachment

T Fast tail movements with body as point of attachment

h Slow body movements with tail as point of attachment

$\mathrm{H} \quad$ Fast body movements with tail as point of attachment

b Slow central body movements with the head of body and tail as a point of attachment

B Fast central body movements with the head of body and tail as a point of attachment

\section{Statistical analyses}

Differences in all parameters measured were tested for between lines using analysis of variance (in a generalised linear modelling procedure) in Minitab Release 14 (Minitab Inc., State College, PA). Experimental line was used as the independent variable. Dependent variables were the behavioural categories (see Table 1) and cercarial produc- tion for chronobiology. Dependent variables were transformed as necessary to meet the generalised model's assumptions of homogeneity of variance and normality of error. Where variables could not be sufficiently transformed, non-parametric Kruskal Wallace tests were used. As our effect sizes are clearly presented, and with appropriate metrics of their precision, we have chosen not to 
incorporate Bonferroni correction procedures here to control type-1 error rate; such procedures are increasingly viewed as unhelpful [25-28].

\section{Results}

\section{Cercarial chronobiology (see figure I)}

The $S$. rodhaini cercariae emerging from single species infections were shown to have an average nocturnal shedding peak of $200 \pm 32$ cercariae per snail between 18302030 hours. A low level of shedding continued throughout the night with an unpredicted small peak of $44 \pm 14$ cercariae per snail between $0630-0830$ hours. S. rodhaini's entire shedding period extended from 1430-1030 hours, however shedding was at a low level $(<50$ cercariae per snail) for all apart from the peak 2 hour period (18302030 hours). In contrast $S$. mansoni cercariae emerging from single species infections had an average diurnal shedding peak of $538 \pm 84$ cercariae per snail (significantly greater than that of $S$. rodhaini $\mathrm{F}_{1,35}=16.64$, $\mathrm{p}<$ 0.001 ) during the period 0830-1030 hours. The time period over which $S$. mansoni shedding occurred was very short 0630-1230 hours with negligible shedding ( $<5$ cercariae per snail) for 4 hours thereafter.

For all snails exposed to both species of schistosome the species of cercaria shedding at each time point were successfully confirmed through genetic sequencing of their mitochondrial cytochrome oxidase subunit 1 (CO1). None of the snails exposed to 3 miracidia of $S$. mansoni and 3 miracidia of $S$. rodhaini simultaneously became patently infected with $S$. rodhaini. Those snails exposed to 6 miracidia of $S$. mansoni and 6 miracidia of $S$. rodhaini and confirmed to only be patently infected with $S$. rodhaini showed a similar emergence pattern to that of individual $S$. rodhaini infections, although the sample size was very small.

Cercariae emerging from snails exposed to $S$. mansoni and S. rodhaini simultaneously at the two different doses, but only patently infected with $S$. mansoni, had lower cercarial production in addition to an apparent slight shift in diurnal rhythms than/from those from $S$. mansoni single species exposures. The peak of shedding that occurred between 0830-1030 hours for snails exposed to and patently infected with $S$. mansoni only was within the same time period for snails exposed to both parasites simultaneously but at a significantly lower level $(176 \pm 24$ cercariae per snail for snails exposed to 3 miracidia of $S$. mansoni and 3 miracidia of $S$. rodhaini simultaneously $\left(\mathrm{F}_{1,43}=\right.$ 25.78, p < 0.01) and $167 \pm 45$ cercariae per snail for snails exposed to 6 miracidia of $S$. mansoni and 6 of $S$. rodhaini simultaneously $\left(\mathrm{F}_{1,22}=9.48, \mathrm{p}=0.006\right)$ ) (Figure 2). A slight shift in the shedding pattern was observed with comparatively high levels of cercarial shedding occurring in the two hours after the peak (1030-1230 hours) (166 \pm 21 cercariae per snail for snails exposed to 3 miracidia of S. mansoni and 3 miracidia of $S$. rodhaini simultaneously and $60 \pm 36$ cercariae per snail for snails exposed to 6 miracidia of $S$. mansoni and 6 of $S$. rodhaini simultaneously) in contrast to the large reduction in cercarial shedding for snails exposed to $S$. mansoni only in the two hours after the peak.

Three snails which were exposed to 6 miracidia of $S$. mansoni and 6 miracidia of $S$. rodhaini were identified to be patently infected with both species using molecular sequencing of the cercariae produced. The peak shedding periods for both species were at the same time (08301030 for S. mansoni and 1830-2030 for S. rodhaini) as for individual infections, however shedding was at lower level for both species from the mixed infection in comparison to single infections $(227 \pm 67$ cercariae per snail for S. mansoni and $53 \pm 33$ cercariae per snail for $S$. rodhaini). The difference was significant for $S$. rodhaini $\left(\mathrm{F}_{1,22}=5.03\right.$, $\mathrm{p}=0.04)$ but not for $S$. mansoni $\left(\mathrm{F}_{1,18}=2.38, \mathrm{p}=0.14\right)$. The time period over which shedding occurred was the same for $S$. mansoni cercariae emerging from mixed infections as for single infections (0630-1230 hours) and the pattern of emergence was similar. Molecular identification of cercariae revealed that the shedding period for $S$. rodhaini cercariae emerging from dually infected snails was restricted in comparison to snails only infected with $S$. rodhaini to 1830-2230 hours, with no shedding throughout the night or further peak in the early morning. This may be due to the significantly lower levels of shedding for $S$. rodhaini and resulted in no overlap in cercarial emergence of the two parasite species from co-infected snails.

\section{Cercarial swimming behaviour}

Cercarial behavioural repertoire; a comparison between S. mansoni and S. rodhaini

Figure 2 gives a comparison of the average percentage of time cercariae from each species spent performing each individual behavioural category when produced from the individually exposed snail groups. No behavioural categories were unique to either species, although the proportion of time spent performing certain categories did differ significantly between the species. As these behavioural categories may not accurately represent those displayed under natural situations, due to the small amount of water in which they were videoed, further analyses were mainly performed on the groupings of behaviours into 'attached' or 'unattached' behavioural categories as defined in Table 1 in order to elucidate differences in the activity levels of the cercaria rather than individual behaviours. S. mansoni cercariae spent an approximately equal amount of time attached as unattached, significantly more time attached than $S$. rodhaini $(H=8.93$, d.f. $=136$, $\mathrm{p}=0.003)$. 
A

S. rodhaini

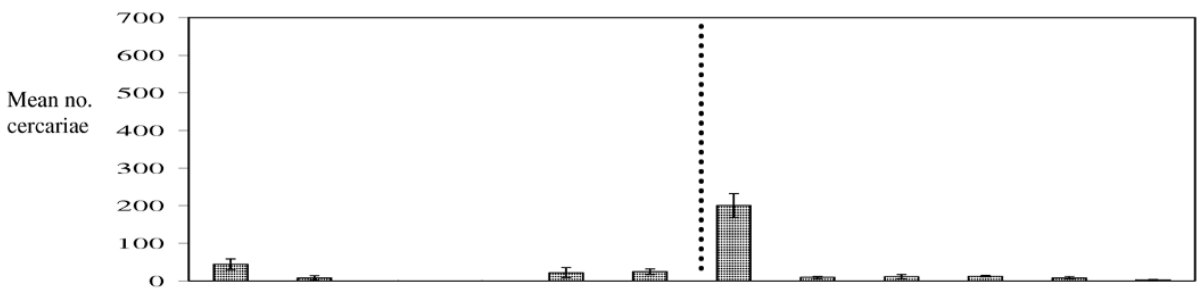

B

S. mansoni

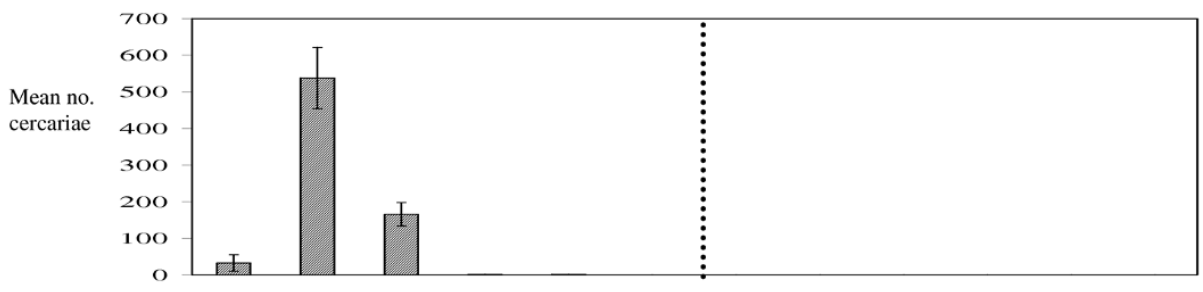

C

Exposed 3 S. mansoni \& 3 S. rodhaini (infected S. mansoni)

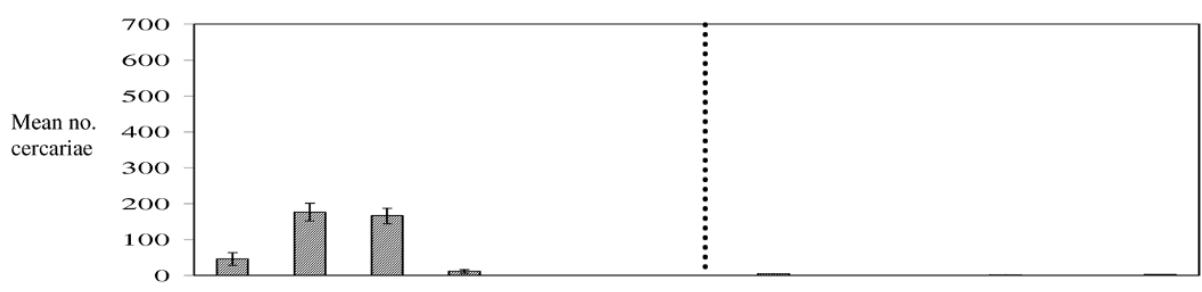

$\mathrm{D}$

Exposed 6 S. mansoni \& 6 S. rodhaini (infected individually)

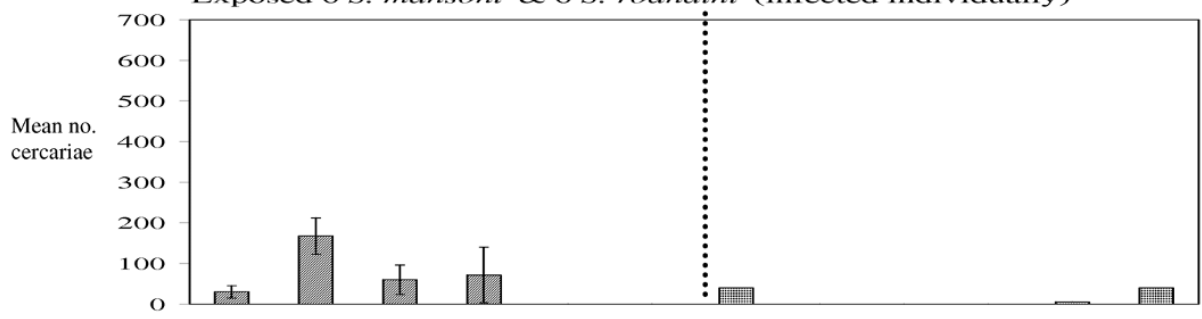

$\mathrm{E}$

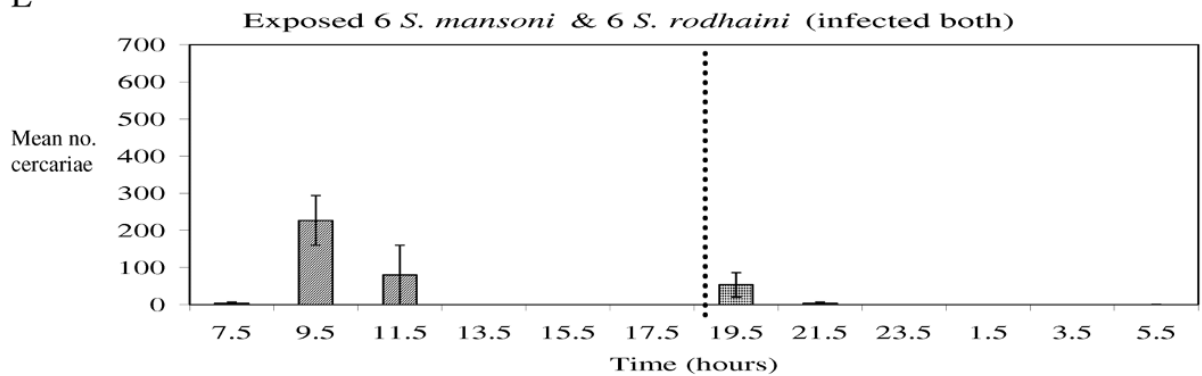

\section{Figure I}

Chronobiology of cercarial emergence. Chronobiology of S. mansoni and S. rodhaini cercarial emergence from snails individually infected with each species (A and $B$ ), snails doubly exposed at a dose of 3 miracidia of each species but only patently infected with S. mansoni (C), doubly exposed at a dose of 6 miracidia of each species but with only single patent infections of $S$. mansoni or S. rodhaini (D) and snails doubly exposed to a dose of 6 miracidia of each species and doubly infected (E) over a 24 hr period (+- SE), ( . . . = transition from light to dark.) 

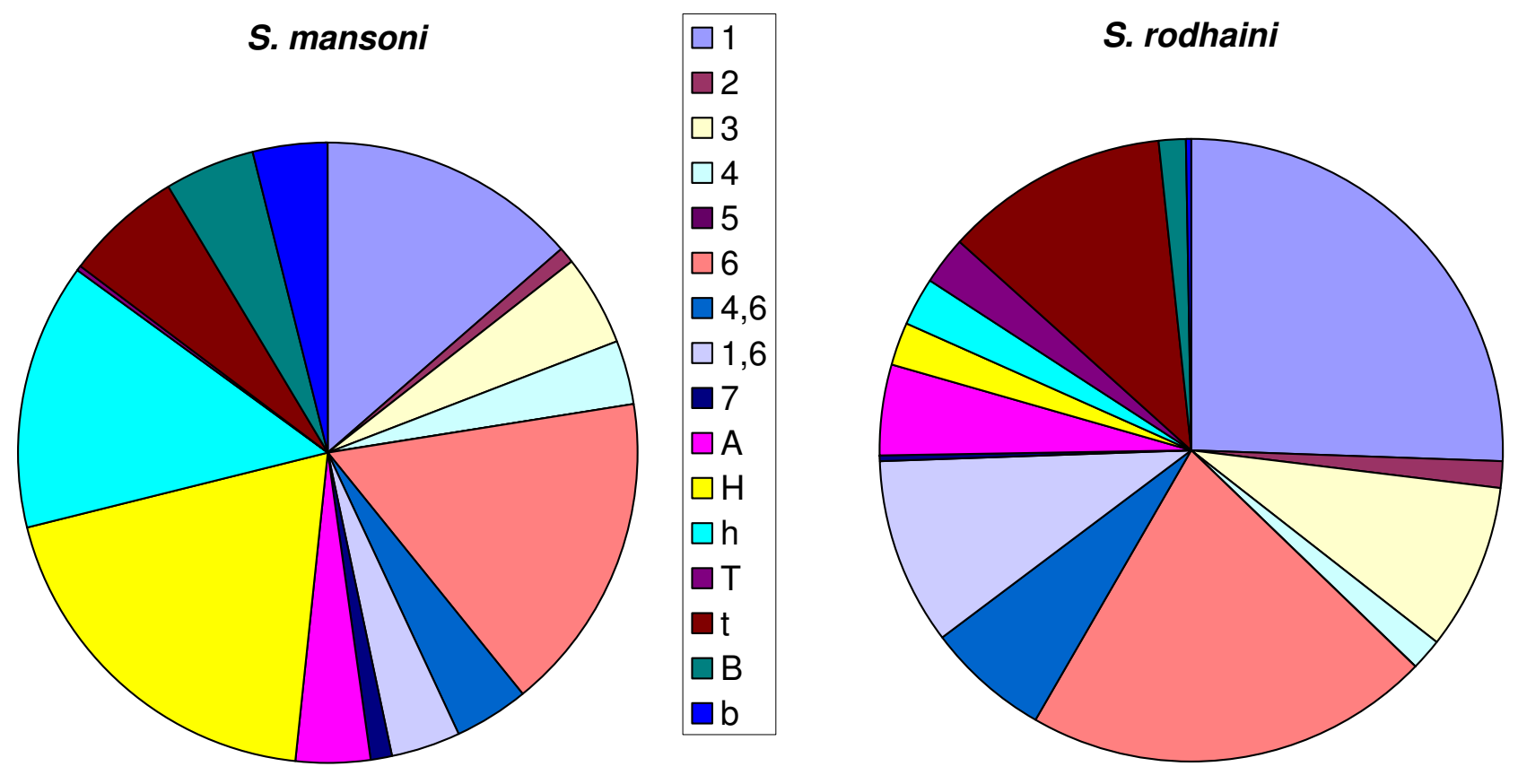

\section{Figure 2}

Cercarial swimming behaviour. Pie charts to show a comparison between the percentage of time unselected S. mansoni and S. rodhaini cercariae spend performing 16 different categories of behaviour (detailed in table I).

The effect of inter-specific competition on cercarial behaviour A significantly greater amount of time was spent performing behaviour '7' (body first swimming) for both species with mixed species conditions $(S$. mansoni $\mathrm{H}=6.65$, d.f. $=$ $160, \mathrm{p}=0.04 ;$ S. rodhaini $\mathrm{H}=30.19$, d.f. $=207, \mathrm{p}<0.001)$.

There was a significant increase in all attached behaviours for $S$. rodhaini under conditions of potential inter-specific competition ( $\mathrm{H}=22.99$, d.f. $=136, \mathrm{p}<0.001$ for ' $\mathrm{H}$ ' (fast head movements), $\mathrm{H}=24.65$, d.f. $=136$, $\mathrm{p}<0.001$ for $\mathrm{h}$ (slow body movements), $\mathrm{H}=10.44$, d.f. $=136, \mathrm{p}=0.005$ for ' $\mathrm{t}$ ' (slow tail movements), $\mathrm{H}=15.73$, d.f. $=136, \mathrm{p}<$ 0.001 for 'B' (fast body movements) and $\mathrm{H}=19.43$, d.f. $=$ $136, \mathrm{p}<0.001$ for ' $\mathrm{b}$ ' (slow body movements)) except for ' $\mathrm{T}$ ' (fast tail movements) where there was no significant difference. For S. mansoni, however, there were no significant changes in attached behaviour with species conditions.

\section{Discussion}

This study has characterized both differences in, and effects of competition on, components of the cercarial phenotype related to host finding and infectivity for two closely related schistosome species. Molecular techniques have enabled, for the first time, subtle effects of inter-specific competition on chronobiology to be revealed and investigation of the cercarial attachment behaviour of both species has identified differences which may affect host finding behaviour.

In addition to confirming the diurnal shedding peak of $S$. mansoni and nocturnal shedding peak of S. rodhaini [17] an extra emergence peak of $S$. rodhaini was also identified in the early morning, consistent with that recently reported from the field in Kenya [16]. This peak could potentially lead to misidentification of $S$. rodhaini infections as $S$. mansoni in the absence of full 24 hour chronobiological investigation or molecular identification of parasites from field collected snails. Additionally, as this has recently been confirmed to be present in natural situations [16], it may lead to an increased likelihood of both parasite species co-infecting the same definitive hosts, allowing hybridization and mating competition between the two species to occur $[9,13,15,16,21]$. We also observed here that the $S$. mansoni shedding peak and indeed overall shedding was greater than that of $S$. rod- 
haini in this B. glabrata intermediate host, indicating that $S$. mansoni was more reproductively successful in this host than $S$. rodhaini.

Inter-specific competition was shown to influence cercarial chronobiology with co-infection resulting in a slight shift in the $S$. mansoni shedding pattern and a reduction of the $S$. rodhaini shedding period (potentially as a result of the observed general reduction in the number of cercaria shed), in comparison to single species infections, preventing overlap and therefore possible interference with $S$. mansoni shedding. Importantly this effect on chronobiology does not result in a large shift for either species and is unlikely therefore to affect the species of hosts available for infection by cercariae on emergence. This effect competition would not have been identified without the use of molecular characterisation of emerging cercariae as it occurred in a period of overlap in shedding of the two species. It demonstrates that although previous studies have shown chronobiology to be genetically determined $[17,18]$ there is also some phenotypic plasticity.

In addition to influencing the time at which cercariae were released, the chronobiological studies revealed that both co-exposure with and without patent co-infection resulted in lower hourly cercarial production for both parasite species in comparison to single species exposures and infections. This reduction was seen for cercariae produced from those snails exposed to three miracidia as well as six of each parasite species simultaneously, therefore excluding miracidial dose as an explanation for the difference in cercarial production and implicating inter-specific competition. Indeed parasite species co-infecting the same host are predicted to undergo competition for shared resources [29] and in this interaction it appears that such competition has negative effects on the asexual reproduction of both the interacting species.

Cercarial activity is known to relate to infectivity $[7,8]$. The main difference observed between swimming behaviours for $S$. mansoni and $S$. rodhaini was that $S$. mansoni cercariae spent a significantly greater amount of time attached to the petri dish than S. rodhaini cercariae. The causality/ functionality of such attachment behaviour is unclear, and of course the possibility of experimental artefact cannot be fully discounted here. However, whether an artefact of this particular laboratory set-up or not, in a previous study time spent attached was shown to be a very useful variable negatively correlated to infectivity to the definitive host [7]. Additionally, the behavioural category identified in this study of 'body first swimming', which $S$. mansoni performed for a significantly greater amount of time than S. rodhaini, has been identified in previous studies where it has been associated with active attachment of a cercaria to a substrate [30]. The lack of post attachment searching behaviour may indicate that such active attachment was performed to allow the cercariae to rest [31], rather than try to penetrate the substrate. The difference in time spent attached between $S$. mansoni and S. rodhaini is therefore likely to represent different strategies of energy conservation between the two species, with $S$. mansoni conserving its energy in the absence of host cues $[5,6]$. No exclusive behavioural categories were identified for either species in this study to allow differentiation between the species through their swimming behaviour. Further investigation in order to directly link phenotypic behaviours of cercariae in natural conditions to the parasite's definitive host species is warranted.

Inter-specific competition was shown to influence these parasite swimming behaviours. The reduction in active swimming behaviours and increase in body first swimming exhibited by both species and increase in attachment time for $S$. rodhaini exhibited under conditions of potential inter-specific competition may reflect reductions in cercarial glycogen levels due to the detrimental effects of inter-specific competition. The lack of difference in amount of time spent attached by $S$. mansoni cercariae emerging from co-exposed snails in comparison to individually exposed snails may indicate that competition between the two parasite species had less of a detrimental effect on S. mansoni than S. rodhaini.

\section{Conclusion}

In conclusion, through investigation of the phenotype of such a readily available and abundant life-stage of the schistosome life-cycle, we have gained important insights into the complexities of this medically important hostparasite system. Inter-specific competition between these schistosome species has been implicated in influencing cercarial production, chronobiology and activity. In natural geographic areas where these two species interact they can be identified with some certainty by comprehensive investigation of their chronobiological shedding peaks, which remain distinct under competition. Further investigation of the effects of inter-specific competition on these phenotypes in the field is now required.

\section{Competing interests}

The authors declare that they have no competing interests.

\section{Authors' contributions}

AJN conducted all the experiments and analyses and wrote the manuscript. DR helped to design the experiments, participated in its coordination and provided substantial revisions to the manuscript. LR analysed the cercarial videos and helped with the writing of the manuscript. JPW led the design of the experiments, provided close supervision throughout and substantial contribution to the manuscript. 


\section{Acknowledgements}

We thank Miss Fiona Allan, Mr Mike Anderson and Mrs Jayne King for assistance with animal husbandry, Mr Richard Kane and Mrs Julia LlewellynHughes for assistance with molecular sequencing and Professor Mike Doenhoff for originally supplying the lines of B. glabrata and S. mansoni used in this investigation. This research was funded by the Wellcome Trust (grant number 067542).

\section{References}

I. King CH, Dickman K, Tisch DJ: Reassessment of the cost of chronic helminthic infection: a meta-analysis of disabilityrelated outcomes in endemic schistosomiasis. Lancet 2005, 365: $1561-1569$.

2. Combes C, Fournier A, Mone H, Theron A: Behaviours in trematode cercariae that enhance parasite transmission: patterns and processes. Parasitology 1994, 109:S3-SI3.

3. Haas W, Haberl B, Kalbe M, Komer M: Snail-host-finding by miracidia and cercariae: Chemical Host Cues. Parasitology Today 1995, II:468-472.

4. Theron A, Mouahid G, Mone H: Schistosoma mansoni : cercarial shedding patterns from a mixed infection of Biomphalaria glabrata with two (early and late) chronobiological variants. Parasitology Research 1997, 83:356-358.

5. Brachs S, Haas W: Swimming behaviour of Schistosoma mansoni cercariae: responses to irradiance changes and skin attractants. Parasitology Research 2008, 102:685-690.

6. Haeberlein S, Haas W: Chemical attractants of human skin for swimming Schistosoma mansoni cercariae. Parasitology Research 2008, 102:657-662.

7. Norton AJ, Blair L, Rollinson D, Webster JP: Schistosoma mansoni cercariae behavioural repertoire and the impact of host genotype and parasite pressure. In Proceedings of Workshop on African Freshwater Malacology, 9-12 September 2003 Edited by: Madsen H. DBL publications; 2007:199-212.

8. Lawson JR, Wilson RA: The relationship between the age of Schistosoma mansoni cercariae and their ability to penetrate and infect the mammalian host. Parasitology 1983, 87:48I-492.

9. Morgan JA, Dejong RJ, Snyder SD, Mkoji GM, Loker ES: Schistosoma mansoni and Biomphalaria: past history and future trends. Parasitology 200I, I23:S2I I-S228.

10. Theron A: Early and late shedding patterns of Schistosoma mansoni cercariae: ecological significance in transmission to human and murine hosts. Journal of Parasitology 1984, 70:652-655.

11. Huygelen C: Treatment of a case of canine bilharziosis due to Schistosoma rodhaini by a derivative of thioxanthone. Ann Soc Belg Med Trop (1920) 1957, 3 I(37):993-997.

12. Schwetz J, Stijns J: Rediscovery of Schistosoma rodhaini Brumpt and discovery of its definitive host. C R Seances Soc Biol Fil 195I, I 45(I5-I6): I 255-I257.

13. Morgan JA, Dejong RJ, Lwambo NJ, Mungai BN, Mkoji GM, Loker ES: First report of a natural hybrid between Schistosoma mansoni and S. rodhaini. Journal of Parasitology 2003, 89:416-418.

14. Pitchford RJ: A check list of definitive hosts exhibiting evidence of the genus Schistosoma Weinland, 1858 acquired naturally in Africa and the Middle East. Journal of Helminthology 1977, 5 I:229-252.

15. Steinauer ML, Mkoji GM, Loker ES: Schistosoma mansoni and Schistosoma rodhaini in western Kenya: a study of species boundaries [abstract]. American journal of tropical medicine and hygiene 2006, 75:212.

16. Steinauer ML, Mwangi IN, Maina GM, Kinuthia JM, Mutuku MW, Agola EL, Mungai B, Mkojo GM, Loker ES: Interactions between natural populations of human and rodent schistosomes in the Lake Victoria region of Kenya: a molecular epidemiological approach. PLoS Negl Trop Dis 2008, 2(4):e222.

17. Theron A: Hybrids between Schistosoma mansoni and S. rodhaini: characterization by cercarial emergence rhythms. Parasitology 1989, 99:225-228.

18. Theron A, Combes C: Genetic analysis of cercarial emergence rhythms of Schistosoma mansoni. Behavioural genetics 1988, 18:201-209.

19. Theron A, Mone H: Shedding patterns of Schistosoma mansoni and Ribeiroia marini cercariae from a mixed infection of Biomphalaria glabrata. Journal of Helminthology 1986, 60:255-259.
20. Mouahid A, Mone H, Chaib A, Theron A: Cercarial shedding patterns of Schistosoma bovis and $S$. haematobium from single and mixed infections of Bulinus truncatus. Journal of Helminthology 1991, 65:8-14.

21. Norton AJ, Webster JP, Kane RA, Rollinson D: Inter-specific parasite competition: mixed infections of Schistosoma mansoni and $S$. rodhaini in the definitive host. Parasitology 2008, 135:473-484.

22. Lewis FA, Stirewalt MA, Souza CP, Gazzinelli G: Large-scale laboratory maintenance of Schistosoma mansoni, with observations of three schistosome/snail host combinations. Journal of Parasitology 1986, 72:813-828.

23. Ulmer MJ: Notes on rearing snails in the laboratory. In Experiments and techniques in Parasitology Edited by: Macinnis AJ, Vogue M. San Francisco: W. H. Freeman and Company; 1970.

24. Stothard JR, Rollinson D: Partial DNA sequences from the mitochondrial cytochrome oxidase subunit I (COI) gene can differentiate the intermediate snail hosts Bulinus globosus and B. nasutus (Gastropoda: Planorbidae). Journal of Natural History 1997, 31 1:727-737.

25. Garcia LV: Escaping the Bonferroni claw in ecological studies. Oikos 2004, 105:657-663.

26. Moran MD: Arguments for rejecting the sequential Bonferroni in ecological studies. Oikos 2003, 100:403-405.

27. Nakagawa S: A farewell to Bonferroni: the problems of low statistical power and publication bias. Behavioural Ecology 2004, 6:1044-1045.

28. Perenger TV: What's wrong with Bonferroni adjustments. British Medical Journal 1998, 316:1236-1238.

29. Esch GW, Curtis LA, Barger MA: A perspective on the ecology of trematode communities in snails. Parasitology 200I, 1 23:S57-S75

30. Haas W, Haberl B, Schmalfuss G, Khayyal M: Schistosoma haematobium cercarial host-finding and host-recognition differs from that of S. mansoni. Journal of Parasitology 1994, 80:345-353.

31. Haas W: Physiological analyses of host-finding behaviour in trematode cercariae: adaptations for transmission success. Parasitology 1994, 109:S15-S29.
Publish with BioMed Central and every scientist can read your work free of charge

"BioMed Central will be the most significant development for disseminating the results of biomedical research in our lifetime."

Sir Paul Nurse, Cancer Research UK

Your research papers will be:

- available free of charge to the entire biomedical community

- peer reviewed and published immediately upon acceptance

- cited in PubMed and archived on PubMed Central

- yours - you keep the copyright

Submit your manuscript here:

http://www.biomedcentral.com/info/publishing_adv.asp
BioMedcentral 